\title{
A Small Case Series of Intravascular Large B-Cell Lymphoma with Unexpected Findings: Subset of Cases with Concomitant Extravascular Central Nervous System (CNS) Involvement Mimicking Primary CNS Lymphoma
}

Kate Poropatich · Dave Dittmann Yi-Hua Chen · Kirtee Raparia Kristy Wolniak · Juehua Gao

Department of Pathology, Northwestern University Feinberg School of Medicine, Chicago, IL, USA

Received: January 19, 2017

Revised: February 13, 2017

Accepted: February 15, 2017

Corresponding Author

Kate Poropatich, MHS, MD

Department of Pathology, Northwestern University

Feinberg School of Medicine, Feinberg Pavilion

7-330, 251 E. Huron St., Chicago, IL 60611, USA

Tel: +1-301-233-3533

Fax: +1-312-503-0189

E-mail: kate.poropatich@northwestern.edu

\begin{abstract}
Background: Intravascular large B-cell lymphoma (IVLBCL) is a rare type of extranodal lymphoma with growth mainly in the lumina of vessels. We studied a small series of IVLBCL and focused on its central nervous system (CNS) involvement. Methods: Searching the medical records of Northwestern Memorial Hospital, we identified five cases of IVLBCL from January 2007 to January 2015. Clinical information, hematoxylin and eosin stained histologic slides and immunohistochemistry studies were reviewed for all cases. Polymerase chain reaction (PCR) analysis for the immunoglobulin (Ig) heavy and light chain gene rearrangement was performed on all five cases. Results: Three of the five cases of IVLBCL were autopsies. Patients' age ranged from 56 to 84 . CNS involvement was present in two cases-in both patients, the CNS involvement showed an extravascular pattern with confluent sheet-like formation. PCR analysis confirmed that in one case the systemic intravascular and CNS extravascular components were clonally identical. Conclusions: In a small case series of IVLBCL, we observed that CNS involvement by IVLBCL often has an extravascular morphology, but is clonally identical to the intravascular counterpart by PCR analysis. As IVLBCL can have a rapidly progressing poor outcome, it should be kept in the differential diagnoses for patients presenting with lymphoma of the CNS. The presence of extravascular growth patterns in the CNS should not exclude IVLBCL as a diagnosis.
\end{abstract}

Key Words: Intravascular large B-cell lymphoma
Intravascular large B-cell lymphoma (IVLBCL) is a rare type of extranodal large B-cell lymphoma characterized by the selective growth of large lymphoma cells within smaller vessel lumina. ${ }^{1}$ Classified as a "Mature B cell" neoplasm in the 2008 World Health Organization (WHO) classification, ${ }^{1}$ IVLBCL is considered phenotypically and morphologically similar to diffuse large B cell lymphoma (DLBCL). However, with less than 500 reported cases, far less is understood about this non-Hodgkin's extranodal lymphoma. With vague clinical symptoms and the absence of lymphadenopathy characteristic of other lymphomas, this entity is thought to be widely underdiagnosed with clinical deterioration occurring rapidly and often resulting in the diagnosis being made at the time of autopsy. ${ }^{2-5}$

IVLBCL is divided into "Western" and "Eastern" variants, with the former showing a high frequency of central nervous system (CNS) and skin involvement and the latter frequently associated with bone marrow (BM) involvement and hemophago- cytic syndrome. ${ }^{6}$ When involving the BM, a predominantly sinusoidal pattern is seen. ${ }^{7}$ The CNS is the most frequent site of involvement in IVLBCL, with over half of all cases presenting with neurologic symptoms and subsequent pathologic evidence of disease in the CNS. ${ }^{8-10}$ It is important to recognize CNS involvement as early as possible in these patients as they have an overall worse clinical outcome and survival rate compared to other sites of involvement such as the skin. ${ }^{10}$

In a small subset of IVLBCL cases there is histomorphologic variability and perivascular involvement has been demonstrated in peripheral organs including the stomach, liver, adrenal glands, bladder, and testis. ${ }^{911-18}$ However, little is known about the histologic heterogeneity of CNS IVLBCL. In particularly aggressive instances involving the $\mathrm{CNS}$, concomitant minimal perivascular and extravascular tumor can be seen. To date, there have been over 10 documented cases of IVLBCL showing an extravascular growth pattern in the CNS, including some cases that presented 
with mass-like lesions on magnetic resonance imaging (MRI)., ${ }^{6,919-21}$ Most striking about these cases is that they mimic patterns seen in primary CNS B-cell lymphoma based on angiocentric growth of large, atypical B cells that have a predilection for the leptomeninges. ${ }^{22}$ It remains unclear what the incidence of this morphologic pattern is in the CNS of patients presenting with IVLBCL.

To further elucidate this question, we studied five cases of IVLBCL and observed two cases with CNS involvement that demonstrated an entirely perivascular and extravascular pattern without an intravascular component. For the first time, we used conventional polymerase chain reaction (PCR) to demonstrate that the extravascular CNS component has the same B-cell clone as the pure intravascular involvement seen in the rest of the organs.

\section{MATERIALS AND METHODS}

\section{Sample characteristics}

We searched medical records of Northwestern Memorial Hospital from January 2007 to January 2015, and identified five cases of IVLBCL that included three autopsies and two living patients. Clinical information was collected. Hematoxylin and eosin-stained diagnostic slides and immunohistochemical stains were reviewed. This study was approved by Northwestern University Institutional Review Board.

\section{Immunohistochemistry}

Immunohistochemical staining was performed on formalinfixed, paraffin-embedded tissue (FFPE). Antigen retrieval and immunohistochemical stains were performed on an automated immunostainer following the manufacturer's protocol (Ventana Medical Systems, Tuscon, AZ, USA). The following predilute antibodies were used: CD3 (Ventana Medical Systems), BCL2 (Cell Marque, Rocklin, CA, USA), BCL6 (Cell Marque), CD20 (Dako, Carpinteria, CA, USA), PAX5 (Cell Marque), CD10 (Leica, Buffalo Grove, IL, USA), and MUM1 (Leica). Determination for germinal center B cell (GCB) and non-GCB was based on expression patterns for CD10, BCL-6, and MUM1 per Hans et al. criteria. ${ }^{23}$ A GCB phenotype was considered if a case was positive for CD10. A non-GCB phenotype was considered with any of the following expression patterns: $\mathrm{CD}^{-} 0^{-}$ $\mathrm{BCL}^{+} \mathrm{MUM}^{+}, \mathrm{CD}^{-} 0^{-} \mathrm{BCL}^{-} \mathrm{MUM}^{+}$, and $\mathrm{CD} 10^{-} \mathrm{BCL}^{-}$ MUM1 $^{-}$. Positive and negative controls were performed with all cases and showed appropriate staining pattern.

\section{Clonality assessment by PCR}

Lymphoma cells were macrodissected from FFPE sections. DNA was quantified by spectrophotometry with NanoDrop 1000 (Thermo Scientific, Waltham, MA, USA). Isolated DNA was tested for gene rearrangements with multiplex PCR using universal primer sets (Invivoscribe Gene Clonality Assays, San Diego, CA, USA) for IgH V-J FR1, FR2, and FR3 primer sets as well as light-chain analysis with $\mathrm{IgK}$ V-J primer sets. $\operatorname{IgK}$ clonality testing utilized two master mixes, Tube A and Tube B, to target conserved regions within variable $(\mathrm{Vk} 1-7)$ and joining regions (Jk1-5). The PCR products were detected by capillary gel electrophoresis on an ABI 3130XI genetic analyzer (Applied Biosystems, Foster City, CA, USA). Dominant peaks were considered to be monoclonal if they were at least three-times the height of the polyclonal background and indeterminate monoclonal cases applied to peaks that were between two-times and three-times the height of the background.

\section{RESULTS}

\section{Clinical features}

From our database search we obtained the records for five patient cases with a diagnosis of IVLBCL, including three autopsy patients and two living patients, both from outside hospitals, who sought a second-opinion diagnosis at our institution (Table $1)$. Three cases were female and two were male. The age range at the time of diagnosis was 56-84 years.

Among autopsy cases, case 1 was an 81-year-old male who suffered from rapidly progressive respiratory failure accompanied by coagulopathy. His laboratories were remarkable for splenomegaly, anemia and thrombocytopenia. He passed away abruptly prior to any further medical work-up and an autopsy was requested. Case 2 was a 76-year-old otherwise healthy female who presented with altered mental status, anemia, fever, hypotension, and renal failure with rapid neurological decompensation, ultimately resulting in death and a subsequent autopsy. Case 3 was an 84-year-old male who presented with three months of fevers and chills and idiopathic thrombocytopenia purpura that did not respond to treatment with intravenous immunglobulin and steroids. Following his death, a limited 'abdomen-only' autopsy was performed.

Cases 4 and 5 were living patients at the time of diagnosis. Case 4 was a 61-year-old female who presented with a primary complaint of 6 months of a progressive skin rash and urticaria accompanied by weakness and intermittent fevers. Laboratory work-up was notable for mild pancytopenia. A skin biopsy was 
Table 1. Summary of clinical and pathologic features of five cases of IVLBCL

\begin{tabular}{|c|c|c|c|c|c|c|}
\hline Case & $\begin{array}{l}\text { Age }(y r) / \\
\text { Sex }\end{array}$ & Clinical presentation & Sites of involvement & Cell of origin & $\begin{array}{l}\text { Bone marrow } \\
\text { involvement }\end{array}$ & Treatment and outcome \\
\hline 1 & $81 / \mathrm{M}$ & Respiratory failure & $\begin{array}{l}\text { Lungs, heart, adrenal glands, } \\
\text { kidneys, prostate, bladder } \\
\text { esophagus, stomach, } \\
\text { pancreas, intestines }\end{array}$ & Non-GCB & No & $\begin{array}{l}\text { None; deceased prior } \\
\text { to diagnosis. }\end{array}$ \\
\hline 2 & $76 / F$ & Encephalopathy & $\begin{array}{l}\text { Brain, skin, breast, heart, lungs, } \\
\text { liver, adrenal glands, stomach, } \\
\text { pancreas, intestines }\end{array}$ & Non-GCB & Yes & $\begin{array}{l}\text { None; deceased prior } \\
\text { to diagnosis }\end{array}$ \\
\hline 3 & $84 / \mathrm{M}$ & Fever and chills & $\begin{array}{l}\text { Adrenal glands, bladder, stomach, } \\
\text { pancreas, intestines, appendix, } \\
\text { liver }\end{array}$ & Non-GCB & No & $\begin{array}{l}\text { IVIG, steroids, deceased } \\
\text { shortly after diagnosis }\end{array}$ \\
\hline 4 & $61 / F$ & $\begin{array}{l}\text { Progressive skin rash; } \\
\text { relapse } 6 \text { mo later presenting } \\
\text { as loss of consciousness }\end{array}$ & Skin, brain & $\begin{array}{l}\text { Initial: non-GCB } \\
\text { Relapse: non-GCB }\end{array}$ & No & $\begin{array}{l}\text { Status post R-CHOP, } \\
\text { R-MPV, aSCT; } \\
\text { disease-free at last } \\
\text { follow-up ( } 22 \text { mo } \\
\text { after CNS recurrence) }\end{array}$ \\
\hline 5 & $56 / F$ & Skin rash & Skin & Non-GCB & No & N/A (outside consultation) \\
\hline
\end{tabular}

IVLBCL, intravascular large B-cell lymphoma; M, male; GCB, germinal center B cell; F, female; IVIG, intravenous immunoglobulin; R-CHOP, rituximab-cytoxan, doxorubicin, vincristine and prednisone; R-MPV, rituximab-methotrexate, procarbazine and vincristine; aSCT, autologous stem cell transplant; CNS, central nervous system; N/A, not available.
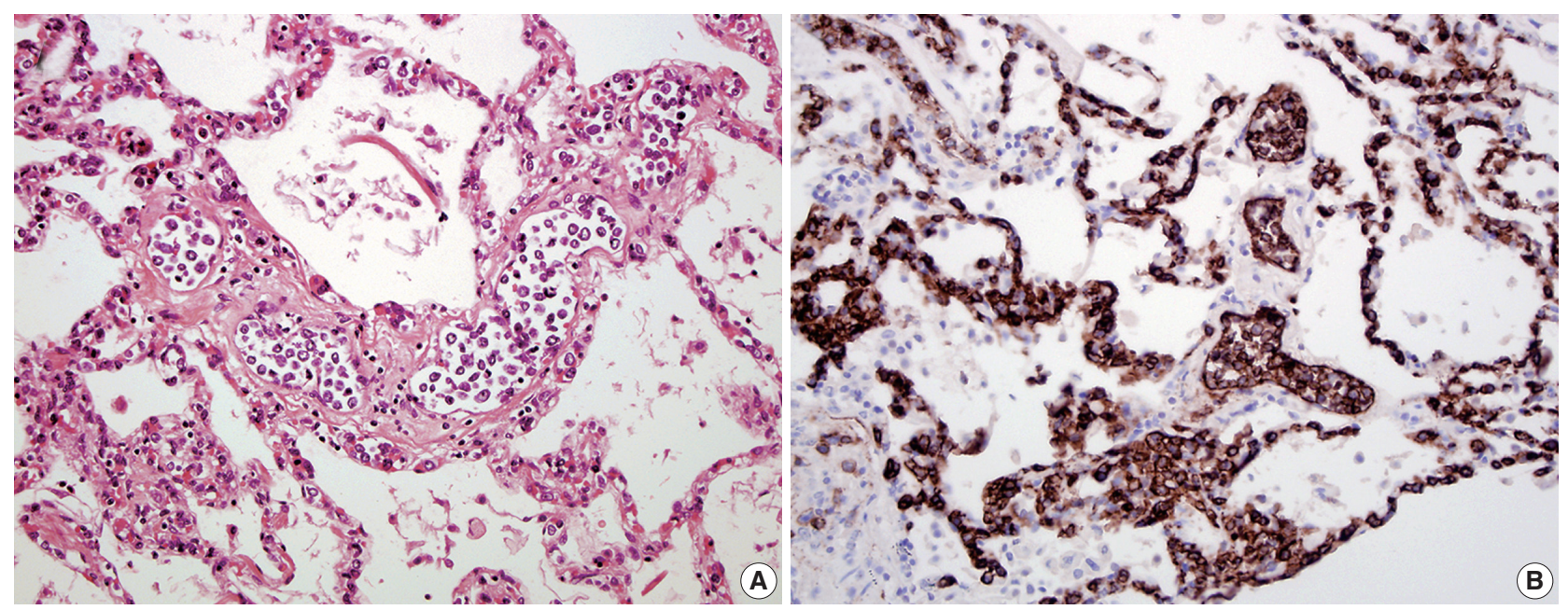

Fig. 1. An 81-year-old male presented with rapidly progressive respiratory failure (case 1). (A) Lung parenchyma showed extensive vascular distension and intravascular collection of large lymphoid cells. (B) A CD20 immunohistochemical stain confirmed many large B cells filling the lumina of the vessels.

performed, which revealed IVLBCL. A BM biopsy was negative for lymphoma. The patient underwent treatment with rituximab-cytoxan, doxorubicin, vincristine and prednisone (R-CHOP) and went into clinical remission. However, 6 months later, the patient presented with sudden loss of consciousness and laboratories remarkable for a markedly elevated lactate dehydrogenase. An MRI was ordered that revealed a $4.5 \mathrm{~cm}$ left frontal brain mass with cystic degeneration. A repeat biopsy of her BM was also performed and was negative for lymphomatous involvement. Following a diagnosis of CNS B-cell lymphoma, she was treated with a new chemotherapy regimen of rituximab-methotrexate, procarbazine and vincristine (R-MPV), intrathecal metho- trexate and autologous stem cell transplantation. She responded positively to the treatment and follow-up MRI's showed resolution of the brain lesion. Case 5 was a 56-year-old female who presented with a history of abdominal skin rash clinically suggestive of acute panniculitis, prompting a skin biopsy of the lesion that was consistent with IVLBCL. A BM biopsy was performed at the time of diagnosis, which was negative for lymphoma.

\section{Histologic, immunophenotypic, and molecular findings}

In all three autopsy cases, intravascular lymphoma cells were present with extensive multi-system involvement as small vessels filled with large cells with scant cytoplasm, vesicular nuclei, and 

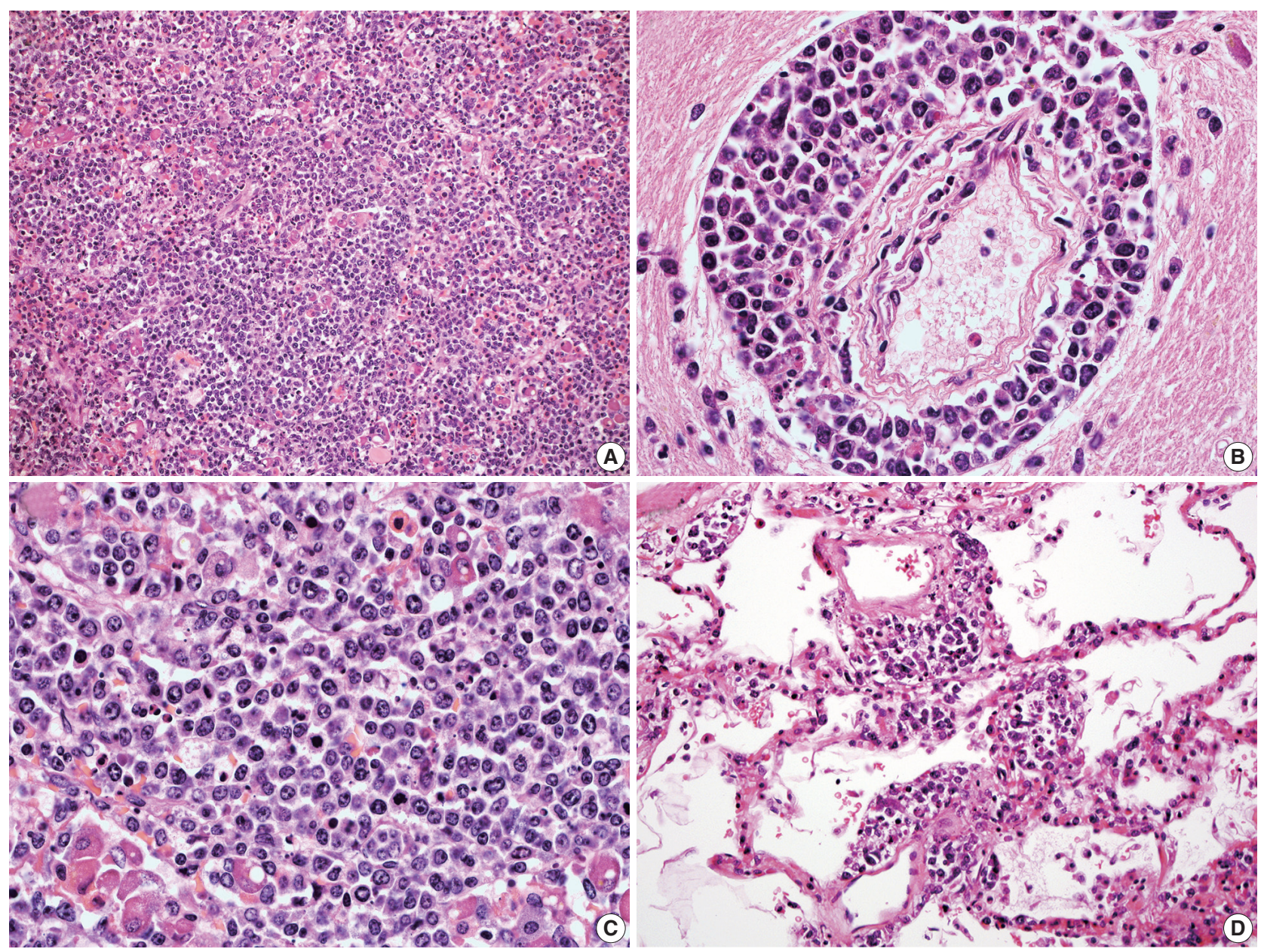

Fig. 2. A 76-year-old female presented with altered mental status and rapid neurologic decompensation (case 2). (A) Histology of the pituitary glands shows perivascular lymphoid infiltrates and areas of sheet-like architecture. (B) Perivascular lymphoid infiltrate cuffing the capillary vessels in the pons. (C) Higher magnification of the pituitary glands reveals sheet-like architecture and parenchymal effacement by abnormal large lymphoid cells with vesicular chromatin and prominent nucleoli. (D) The large lymphoma cells in the intravascular spaces of the lung interstitium from the same patient.

prominent nucleoli. In case 1, histology showed intravascular lymphomatous involvement of multiple organs including the lungs, heart, adrenal glands, esophagus, stomach, pancreas, intestines, kidneys, prostate, and bladder. The largest tumor burden was within the small vessels of the lung interstitium. A CD20 immunostain confirmed that the vessels were distended and filled with large B cells (Fig. 1). A brain dissection was not performed. In case 2, multiple organs were also involved, which included the brain, skin, breast, heart, lungs, liver, adrenal glands, stomach, pancreas, and intestines. The largest tumor burden was observed in the brain, including the cerebral hemispheres, meninges, midbrain, pons and basal ganglia, which demonstrated purely perivascular growth. The pituitary was also involved, and it showed near total effacement of the parenchyma with large, solid sheets of these cells in a sheet-like architecture with an identical morphology to the perivascular component in the other locations in the brain as well as the intravascular component seen in other organs (Fig. 2). A BM biopsy showed intrasinusoidal infiltration of neoplastic B cells. Case 3 revealed predominantly intravascular involvement of large $\mathrm{B}$ cells in adrenal glands, bladder, stomach, pancreas, intestines, appendix, and liver. A brain dissection was not performed.

In cases 4 and 5, skin biopsies revealed large B cells with irregular nuclear contours and prominent nucleoli filling small vessels of the dermis. The patient in case 4 underwent a lumbar puncture after developing neurologic symptoms with a brain mass in the left frontal cortex on MRI, that showed rare atypical large B cells in the cerebrospinal fluid. A biopsy of the brain lesion revealed atypical B cells in the superficial subcortical brain parenchyma organized in sheets and with clustering around 
small vascular spaces, without discernible intravascular involvement (Fig. 3).

For all cases, the cell of origin for the neoplastic large cells was consistent with the non-GCB phenotype according to the Hans et al. algorithm. ${ }^{23}$ More specifically, the large B cells in cases $1-4$ were $\mathrm{CD}^{-} \mathrm{BCL}^{-} \mathrm{MUM}^{+}$; and the large $\mathrm{B}$ cells in case 5 showed $\mathrm{CD} 10{ }^{-} \mathrm{BCL} 6^{+} \mathrm{MUM}^{+}$staining pattern. In case 2 , the neoplastic cells from the extravascular CNS involvement had the same non-GCB phenotype as the intravascular component.

Immunoglobulin gene rearrangement studies were performed
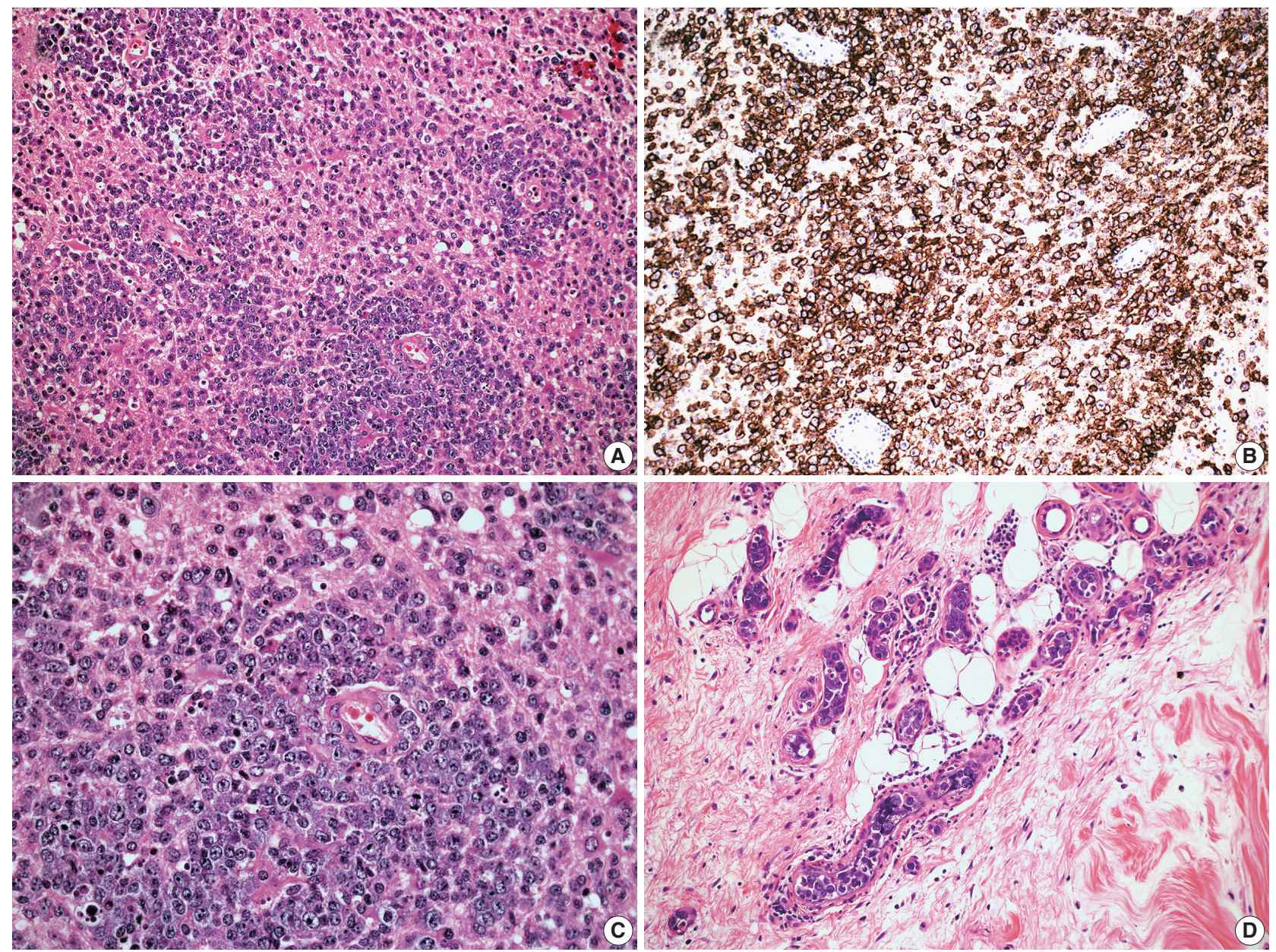

Fig. 3. A 61-year-old female with progressive skin rash and a diagnosis of intravascular large B-cell lymphoma who subsequently developed neurologic symptoms (case 4). (A) Brain biopsy (left frontal cortex) reveals lymphoid infiltrates in predominantly extravascular pattern. (B) CD20 highlights large B cells in the perivascular infiltrates. (C) Brain biopsy reveals sheets of large cells with vesicular chromatin and prominent nucleoli. (D) Diagnostic skin biopsy reveals large lymphoma cells filling the lumina of capillary vessels in the dermis in the same patient.

Table 2. Immunoglobulin heavy chain and light chain gene rearrangement analysis for five cases of IVLBCL

\begin{tabular}{|c|c|c|c|}
\hline & \multicolumn{3}{|c|}{ Immunoglobulin gene rearrangement by PCR } \\
\hline & $\lg \mathrm{H}$ & $\operatorname{lgK}($ tube A) & $\operatorname{lgK}($ tube B) \\
\hline Case 1 & Negative & Positive (272 bp) & Positive (239 bp) \\
\hline Case 2 & Positive (319 bp, 254 bp) & Positive (149 bp, 201 bp) & Positive (275 bp) \\
\hline CNS: meninges & Negative & Positive (149 bp, 201 bp) & Positive (275 bp) \\
\hline CNS: pituitary & Positive (254 bp) & Negative & Negative \\
\hline Case 3 & Negative & Negative & Negative \\
\hline Case 4 & Negative & Negative & Negative \\
\hline Case 5 & Negative & Positive (149 bp) & Positive (237 bp) \\
\hline
\end{tabular}

IVLBCL, intravascular large B-cell lymphoma; PCR, polymerase chain reaction; CNS, central nervous system. 


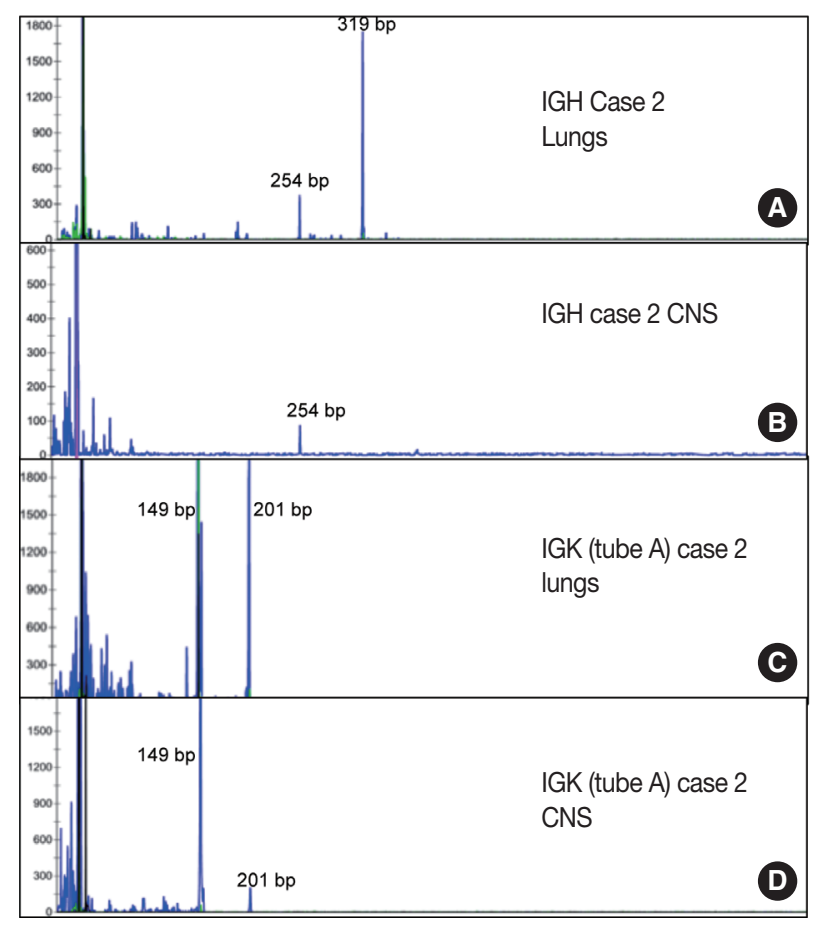

Fig. 4. Immunoglobulin heavy and light chain gene rearrangement performed on tumor cells with two distinct morphologic patterns (case 2). (A, B) Both the lung with intravascular pattern and central nervous system (CNS) pituitary with extravascular pattern show clonal heavy chain rearrangement with the same 254-bp clonal FR2 peak. (C, D) Both the lung with intravascular pattern and CNS meninges with extravascular pattern show clonal kappa light chain rearrangement with the same 149-bp and 201-bp clonal peaks.

in all five cases. Immunoglobulin heavy chain was clonally rearranged in one of the five cases (case 2). Kappa light chain gene rearrangement analysis provided evidence of clonality in two additional cases (cases 1 and 5) (Table 2). To confirm the clonal relationship between the extravascular and intravascular components, immunoglobulin heavy and light chain gene rearrangement studies were performed in case 2 from areas with two distinct morphologies. The pituitary gland, which showed sheet-like extravascular component, was positive for clonal heavy chain gene rearrangement with a 254-bp clonal FR2 peak, identical to one of the clonal peaks detected in the intravascular component from the lung parenchyma. Similarly, the perivascular lymphoid infiltrate from the meninges was positive for clonal kappa light chain rearrangement, with a clonal peak identical to those detected in the intravascular component from the lung parenchyma (Table 2, Fig. 4).

\section{DISCUSSION}

In this study, we reported five cases of IVLBCL, comprised of three autopsy cases and two living patient cases. In all three autopsy cases, the patients died before or shortly after the diagnosis was established. All cases demonstrated extensive organ involvement by the lymphoma cells. Not surprisingly, we observed that IVLBCL is a highly aggressive entity with nonspecific clinical findings and where other differentials are more commonly exhausted, which delays the diagnosis and leads to unsalvageable clinical outcomes. In keeping with past findings, ${ }^{6,13,24}$ our findings confirm that IVLBCL is predominantly non-germinal center B cell in origin as all five cases in this study showed this phenotype.

The CNS is the most common site to be involved by IVBCLup to two-thirds of all cases of IVLBCL—and is a frequent site of intra-organ recurrence. ${ }^{8-10}$ Approximately one-half of all cases of IVLBCL involving the brain are diagnosed post-mortem. ${ }^{25}$ Diagnosing IVLBCL premortem in the setting of CNS involvement is difficult because patients often present with non-specific encephalopathic symptoms, and less frequently stroke-like symptoms including extremity paralysis. ${ }^{10}$ Among living patients diagnosed with CNS-IVLBCL, a subset of cases_-approximately one-third — have been found to survive to 2 years following initial diagnosis. ${ }^{10}$ In our small series of five IVLBCL, two patients developed neurologic symptoms. Both showed biopsy-proven CNS involvement of IVLBCL. Interestingly, in both cases, the CNS involvement was exclusively extravascular characterized by perivascular infiltrate of the abnormal large lymphocytes or confluent sheet like growth. It remains unknown how common the extravascular morphology is in the CNS of IVLBCL patients. In one study, two cases had CNS IVLBCL with a predominant angiocentric pattern," accounting for one-third of the cases in that study. In a separate study of five cases of CNS IVLBCL, all five had predominantly extravascular parenchymal involvement. ${ }^{19}$ Albeit these were small cohorts of cases, extravascular extension into the CNS parenchyma by IVLBCL is probably a more common phenomenon than currently appreciated.

When presenting with primarily CNS symptoms, IVLBCL can mimic CNS B-cell lymphoma and fail to tip off clinicians to look for a more systemic lymphomatous component. Primary CNS B-cell lymphoma, also a rare variant of DLBCL, has a proclivity to show angiocentric proliferation of neoplastic B cells. ${ }^{26}$ In one instance, the perivascular growth pattern in the CNS in a patient with established IVLBCL resulted in an entirely separate diagnosis of primary CNS lymphoma being made, potentially creating unnecessary confusion. ${ }^{6}$ Our findings raise the importance of keeping IVLBCL in the differential of primary CNS lymphomas and that the presence of perivascular growth 
patterns should not exclude IVLBCL as a diagnosis. It is important to identify CNS involvement in patients with IVLBCL as they have a worse clinical outcome than those without CNS involvement and require closer clinical monitoring. ${ }^{10}$

In our series, both cases 4 and 5 presented with cutaneous involvement as their initial manifestation of IVLBCL, raising the possibility of a "cutaneous variant." The "cutaneous variant" is thought to occur preferentially in females and make up a sizeable subset of all IVLBCL cases (up to $26 \%$ in one study of 38 patients), in which the only symptomatology is in the form of a skin rash or plaques, without other sites of involvement. ${ }^{27} \mathrm{Up}$ to one-third of patients with cutaneous presentation may go on to develop other sites of involvement, such as the CNS (as was the case for our case 4), but these cases do not fall under the 'cutaneous variant' subset. In keeping with findings in the literature that the patients with cutaneous involvement have improved survival, case 4 in our series was disease-free at 22 months following their CNS relapse. Case 5 received their clinical treatment outside of our institution and clinical follow-up could not be assessed.

Some challenge the notion of IVLBCL concomitantly involving the CNS extravascularly due to the fact that they are thought to have absent surface adhesion molecules CD29, CD54 (ICAM-1), and CD11a, preventing them from being able to cross small CNS vessels to form perivascular or extravascular infiltrates. ${ }^{28}$ However, a biologic explanation for the predilection for IVLBCL to involve the CNS with an extravascular growth pattern could be due to the fact that as veno-occlusive events occur secondary to small vessel involvement by IVLBCL, the permeability of the blood-brain-barrier is altered ${ }^{29}$ such that it allows for passive diffusion of neoplastic cells across vessels into the brain parenchyma to form extravascular infiltrates.

A diagnosis of IVLBCL is made based on the clinical features and histologic findings of selective intravascular growth of large $\mathrm{B}$ cells. Clonal rearrangement of immunoglobulin chains is not required for the diagnosis. In fact, the sensitivity of PCR-based clonality assays is not very high in large B-cell lymphomas. In our small series, only one case was positive for clonal heavy chain gene rearrangement. Using a combination of heavy and light chain approach, we were able to expand molecular evidence of clonality in three of the five cases.

Thus, in cases when two different histologies coexist in the same patient, molecular modalities are useful to determine the clonal relationship between distinct growth-patterns. Here, we used a molecular approach to confirm that CNS-involvement with extravascular morphology was clonally identical to the intravascular component in the same patient. To our knowledge, this is the first study using conventional molecular diagnostic modalities to demonstrate that extravascular B cells in the CNS of a patient are clonally identical to systemic intravascular cells in the same patient with IVLBCL. Rare past efforts have failed, as indeed, it is difficult to work with such scant material for molecular studies. ${ }^{19}$

In summary, of the two of five cases in this study with CNS involvement, both show pure perivascular and extravascular growth, which in combination with other reported cases in the literature, raises the possibility that CNS involvement is more frequently extravascular in IVLBCL than currently appreciated. Therefore, it is the intent of this study to raise awareness that IVLBCL is not just a clinically heterogenous entity but is also a histologically heterogenous entity with potential proclivity to form extravascular foci in the CNS due to biologically unique properties of the blood brain barrier. Further analysis is needed, particularly in the form of large retrospective cohort studies of known IVLBCL patients, to pinpoint the frequency of extravascular morphology in the CNS in these patients.

\section{Conflicts of Interest}

No potential conflict of interest relevant to this article was reported.

\section{REFERENCES}

1. Campo E, Swerdlow SH, Harris NL, Pileri S, Stein H, Jaffe ES. The 2008 WHO classification of lymphoid neoplasms and beyond: evolving concepts and practical applications. Blood 2011; 117: 501932.

2. Murase T, Yamaguchi M, Suzuki R, et al. Intravascular large B-cell lymphoma (IVLBCL): a clinicopathologic study of 96 cases with special reference to the immunophenotypic heterogeneity of CD5. Blood 2007; 109: 478-85.

3. Fulling KH, Gersell DJ. Neoplastic angioendotheliomatosis: histologic, immunohistochemical, and ultrastructural findings in two cases. Cancer 1983; 51: 1107-18.

4. Ip M, Chan KW, Chan IK. Systemic inflammatory response syndrome in intravascular lymphomatosis. Intensive Care Med 1997; 23: 783-6.

5. Devlin T, Moll S, Hulette C, Morgenlander JC. Intravascular malignant lymphomatosis with neurologic presentation: factors facilitating antemortem diagnosis. South Med J 1998; 91: 672-6.

6. Ponzoni M, Ferreri AJ, Campo E, et al. Definition, diagnosis, and 
management of intravascular large B-cell lymphoma: proposals and perspectives from an international consensus meeting. J Clin Oncol 2007; 25: 3168-73.

7. Estalilla OC, Koo CH, Brynes RK, Medeiros LJ. Intravascular large B-cell lymphoma: a report of five cases initially diagnosed by bone marrow biopsy. Am J Clin Pathol 1999; 112: 248-55.

8. Fonkem E, Lok E, Robison D, Gautam S, Wong ET. The natural history of intravascular lymphomatosis. Cancer Med 2014; 3: 1010-24.

9. Glass J, Hochberg FH, Miller DC. Intravascular lymphomatosis: a systemic disease with neurologic manifestations. Cancer 1993; 71: 3156-64

10. Fonkem E, Dayawansa S, Stroberg E, et al. Neurological presentations of intravascular lymphoma (IVL): meta-analysis of 654 patients. BMC Neurol 2016; 16: 9.

11. Wick MR, Mills SE, Scheithauer BW, Cooper PH, Davitz MA, Parkinson K. Reassessment of malignant "angioendotheliomatosis": evidence in favor of its reclassification as "intravascular lymphomatosis". Am J Surg Pathol 1986; 10: 112-23.

12. Willemze R, Kerl H, Sterry W, et al. EORTC classification for primary cutaneous lymphomas: a proposal from the Cutaneous Lymphoma Study Group of the European Organization for Research and Treatment of Cancer. Blood 1997; 90: 354-71.

13. Yegappan S, Coupland R, Arber DA, et al. Angiotropic lymphoma: an immunophenotypically and clinically heterogeneous lymphoma. Mod Pathol 2001; 14: 1147-56.

14. Podduturi V, Guileyardo JM, Soto LR, Krause JR. A case series of clinically undiagnosed hematopoietic neoplasms discovered at autopsy. Am J Clin Pathol 2015; 143: 854-60.

15. Thomas CA, Guileyardo JM, Krause JR. An intravascular lymphoma with extravascular tendencies. Proc (Bayl Univ Med Cent) 2014; 27: 341-3.

16. Demirer T, Dail DH, Aboulafia DM. Four varied cases of intravascular lymphomatosis and a literature review. Cancer 1994; 73: 1738-45.

17. Case records of the Massachusetts General Hospital. Weekly clinicopathological exercises. Case 39-1986. A 66-year-old woman with fever, fluctuating neurologic signs, and negative blood cultures. N Engl J Med 1986; 315: 874-85.

18. Ferry JA, Harris NL, Picker LJ, et al. Intravascular lymphomatosis (malignant angioendotheliomatosis): a B-cell neoplasm expressing surface homing receptors. Mod Pathol 1988; 1: 444-52.
19. Imai H, Kajimoto K, Taniwaki M, et al. Intravascular large B-cell lymphoma presenting with mass lesions in the central nervous system: a report of five cases. Pathol Int 2004; 54: 231-6.

20. Takeuchi Y, Hashizume Y, Hoshiyama M, Hirose Y, Takahashi A. An autopsy case of intravascular malignant lymphomatosis with intracranial lymphomatous mass lesions. Rinsho Shinkeigaku 1995; 35: 158-63.

21. Beal MF, Fisher CM. Neoplastic angioendotheliosis. J Neurol Sci 1982; 53: 359-75.

22. Fonseca VR, Espada E, Geraldes R, et al. Synchronous brain and intravascular B-cell lymphoma after remission of an adult hemophagocytic syndrome. Clin Case Rep 2016; 4: 327-30.

23. Hans CP, Weisenburger DD, Greiner TC, et al. Confirmation of the molecular classification of diffuse large B-cell lymphoma by immunohistochemistry using a tissue microarray. Blood 2004; 103: $275-82$.

24. Murase T, Nakamura S, Kawauchi K, et al. An Asian variant of intravascular large B-cell lymphoma: clinical, pathological and cytogenetic approaches to diffuse large B-cell lymphoma associated with haemophagocytic syndrome. Br J Haematol 2000; 111: 826-34.

25. Baehring JM, Longtine J, Hochberg FH. A new approach to the diagnosis and treatment of intravascular lymphoma. J Neurooncol 2003; 61: 237-48.

26. Sugita $Y$, Muta $H$, Ohshima K, et al. Primary central nervous system lymphomas and related diseases: Pathological characteristics and discussion of the differential diagnosis. Neuropathology 2016; 36: 313-24

27. Ferreri AJ, Campo E, Seymour JF, et al. Intravascular lymphoma: clinical presentation, natural history, management and prognostic factors in a series of 38 cases, with special emphasis on the 'cutaneous variant'. Br J Haematol 2004; 127: 173-83.

28. Kinoshita M, Izumoto S, Hashimoto $\mathrm{N}$, et al. Immunohistochemical analysis of adhesion molecules and matrix metalloproteinases in malignant CNS lymphomas: a study comparing primary CNS malignant and CNS intravascular lymphomas. Brain Tumor Pathol 2008; 25: 73-8

29. Weiss N, Miller F, Cazaubon S, Couraud PO. The blood-brain barrier in brain homeostasis and neurological diseases. Biochim Biophys Acta 2009; 1788: 842-57. 